\title{
A Differencing Algorithm for Object-Oriented Programs
}

\author{
Taweesup Apiwattanapong, Alessandro Orso, and Mary Jean Harrold \\ Georgia Institute of Technology \\ Atlanta, Georgia \\ E-mail: $\{$ term|orso|harrold\}@cc.gatech.edu
}

\begin{abstract}
During software evolution, information about changes between different versions of a program is useful for a number of software engineering tasks. For many of these tasks, a purely syntactic differencing may not provide enough information for the task to be performed effectively. This problem is especially relevant in the case of object-oriented software, for which a syntactic change can have subtle and unforeseen effects. In this paper, we present a technique for comparing object-oriented programs that identifies both differences and correspondences between two versions of a program. The technique is based on a representation that handles object-oriented features and, thus, can capture the behavior of object-oriented programs. We also present JDIFF, a tool that implements the technique for Java programs, and empirical results that show the efficiency and effectiveness of the technique on a real program.
\end{abstract}

\section{Introduction}

Software maintenance tasks often involve analyses of two versions of a program: an original version and a modified version. Many program-analysis tools that automate these tasks require knowledge of the locations of changes between the two program versions. In addition, some tools also need the mapping of entities, such as statements and methods, from the original version to their counterparts in the modified version. Differencing algorithms can provide information about the locations of changes and can classify program entities as added, deleted, modified, or unchanged.

The results of these differencing algorithms are therefore useful for many software-maintenance tasks. For example, for program-profile estimation (also referred to as stale profile propagation [15]), the differencing results, along with the coverage or profile information for the original version of the program, are used to estimate the coverage or profile information for the modified version. This approach eliminates the cost of rerunning the test suite on the modified version of the program to measure coverage or to obtain profiles. The approach also facilitates the estimation of coverage or profile information in cases in which this information cannot be reproduced (e.g., coverage/profiles from deployed software). For another example, the change information that is produced by differencing is also useful for impact analysis and regression testing. Impact analysis identifies the parts of a program that are affected by changes and, thus, requires knowledge of the location of such changes. Many regression test selection techniques (e.g., $[11,13])$ use change information to select test cases to be rerun on modified versions of the software. For yet another example, in collaborative environments, differencing information is used for merging two modified versions of a software system into a new version that includes the changes from both earlier versions [8].

There are a number of existing techniques and tools for computing textual differences between files (e.g., the UNIX diff utility [9]). However, these techniques are limited in their ability to detect differences in programs because they provide purely syntactic differences and do not consider changes in program behavior indirectly caused by syntactic modifications.

Consider, for example, the two partial Java programs in Figure 1: the original program $P$ and the modified version $P^{\prime}$. If we were to inspect the output of diff, run on $P$ and $P^{\prime}$, we would see that method B.m1 has been added in $P^{\prime}$ and that the exception-type hierarchy has changed. However, without additional analyses, it would not be straightforward to detect that, in $P$ and $P^{\prime}$, the call to a.m1 in D.m3 can be bound to different methods, and the exception thrown in D.m3 can be caught by a different catch block.

Other existing differencing techniques are specifically targeted at comparing two versions of a program (e.g., [4, $6,15])$, but they are not suitable for object-oriented code. BMAT [15] cannot recognize, for instance, differences caused by changes in the exception hierarchy, and would overlook that the exception thrown in D.m3 can be caught by different catch blocks in $P$ and $P^{\prime}$. Semantic diff [6] is defined to work only at the procedure level and cannot be straightforwardly extended to work on entire object-oriented programs. Horwitz's technique [4] is also not suitable for object-oriented programs, in that it is defined only for a simplified C-like language. 


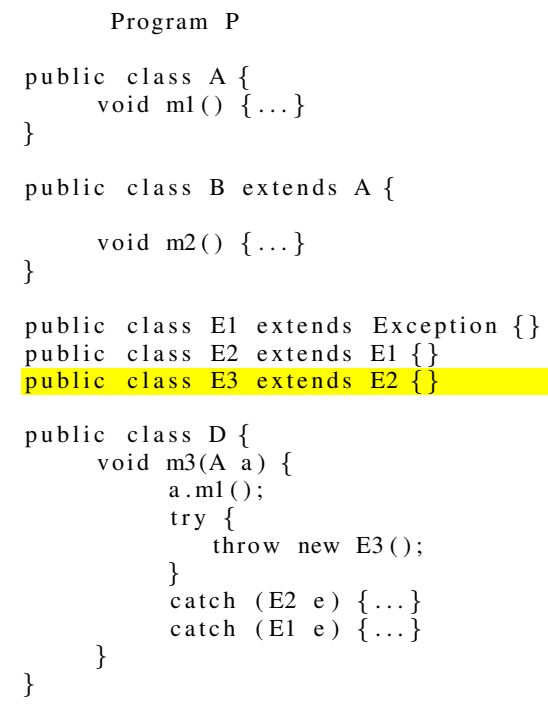

Figure 1. Partial code for an original program $(P)$ and the corresponding modified version $\left(P^{\prime}\right)$.

To overcome problems with existing approaches and provide the differencing information required for tasks such as program-profile estimation, impact analysis, and regression testing, we defined a new graph representation and a differencing algorithm that uses the representation to identify and classify changes at the statement level between two versions of a program. Our representation augments a traditional control-flow graph $(\mathrm{CFG})^{1}$ to model behaviors in the program due to object-oriented features. Using this graph, we identify changes in those behaviors and relate them to the point of the code where the different behavior occurs.

Our algorithm extends an existing differencing algorithm [7] and consists of five steps. First, it matches classes, interfaces, and methods in the two versions. Second, it builds enhanced CFGs for all matched methods in the original and modified versions of the program. Third, it reduces all graphs to a series of nodes and single-entry, singleexit subgraphs called hammocks. Fourth, it compares, for each method in the original version and the corresponding method in the modified version, the reduced graphs, to identify corresponding hammocks. Finally, it recursively expands and compares the corresponding hammocks.

The main contributions of the paper are:

- A new graph representation that models the behavior of object-oriented programs.

- A differencing algorithm that works on the graph representations and uses different heuristics to increase the precision of the results.

- A set of empirical studies that show the efficiency and precision of an implementation of our algorithm.

\footnotetext{
${ }^{1}$ A control-flow graph is a directed graph in which nodes represent statements and edges (possibly labeled) represent flow of control between statements.
}

\section{Differencing Algorithm}

In this section, we first overview the algorithm. Then, we detail the levels at which the algorithm compares the original and modified versions of the program. Finally, we discuss the algorithm's complexity.

\subsection{Overview}

Our algorithm, CalcDiff, given in Figure 2, takes as input an original version of a program $(P)$ and a modified version of that program $\left(P^{\prime}\right)$. The algorithm also inputs two parameters, which are used in the node-level matching. Parameter $L H$ is the maximum lookahead that CalcDiff uses when attempting to match nodes in methods. Parameter $S$ is used when determining the similarity of two hammocks. At completion, the algorithm outputs a set of pairs $(N)$ in which the first element is a pair of nodes and the second element is the status - either "modified" or "unchanged." The algorithm also returns sets of pairs of matching classes $(C)$, interfaces $(I)$, and methods $(M)$ in $P$ and $P^{\prime}$.

CalcDiff performs its comparison first at class and interface levels, then at the method level, and finally at the node level. The algorithm first compares each class in $P$ with the like-named class in $P^{\prime}$, and each interface in $P$ with the like-named interface in $P^{\prime}$, and produces sets of class pairs $(C)$ and interface pairs $(I)$, respectively. For each pair of classes and interfaces, CalcDiff then matches methods in the class or interface in $P$ with methods having the same signature in the class or interface in $P^{\prime}$; the result is a set of method pairs $(M)$. Finally, for each pair of concrete (i.e., not abstract) methods in $M$, the algorithm constructs enhanced CFGs (hereafter, ECFGs) for the two methods and match nodes in the two ECFGs.

The next sections give details of CalcDiff, using the code in Figure 1 as an example. 


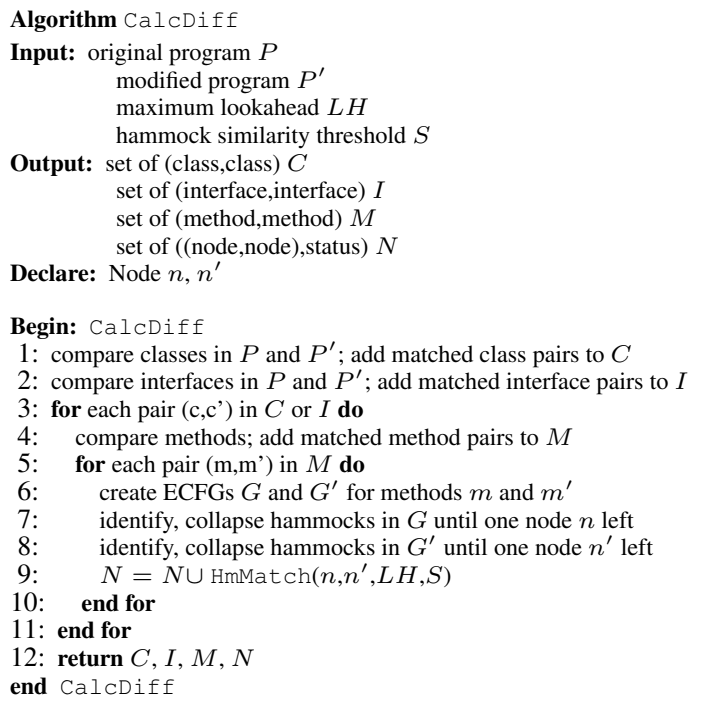

Figure 2. Algorithm CalcDiff

\subsection{Class and Interface Levels}

CalcDiff begins its comparison at the class and interface levels (lines 1-2). The algorithm matches classes (resp., interfaces) that have the same fully-qualified name; the fully-qualified name consists of the package name followed by the class or interface name. Matching classes (resp., interfaces) in $P$ and $P^{\prime}$ are added to $C$ (resp., $I$ ). Classes in $P$ that do not appear in set $C$ are deleted classes, whereas classes in $P^{\prime}$ that do not appear in set $C$ are added classes. Analogous considerations hold for interfaces. In the example programs in Figure 1, each class in $P$ has a match in $P^{\prime}$, and, thus, there is a pair in $C$ for each class in $P$.

\subsection{Method Level}

After matching classes and interfaces, CalcDiff compares, for each pair of matched classes or interfaces, their methods (lines 3-4). The algorithm first matches each method in a class or interface with the method with the same signature in another class or interface. Then, if there are unmatched methods, the algorithm looks for a match based on the name only. This matching accounts for cases in which parameters are added to (or removed from) an existing method, which we found to occur in practice, and increases the number of matches at the node level. Pairs of matching methods are added to $M$. Like for classes, methods in $P$ that do not appear in set $M$ are deleted methods, whereas methods in $P^{\prime}$ that do not appear in set $M$ are added methods. In the example (Figure 1), there would be a pair in $M$ for each method in $P$, but not for method B.m1 in $P^{\prime}$ (which would therefore be considered as added).

\subsection{Node Level}

CalcDiff uses the sets of matched method pairs $(M)$ to perform matching at the node level. First, the algorithm considers each pair of matched methods $\left\langle m, m^{\prime}\right\rangle$ in $M$, and builds ECFGs $G$ and $G^{\prime}$ for $m$ and $m^{\prime}$ (lines 5-6). Then, the algorithm identifies all hammocks in $G$ and $G^{\prime}$, and collapses $G$ and $G^{\prime}$ to one node (lines 7-8); we call these nodes $n$ and $n^{\prime}$, respectively. Next, CalcDiff calls procedure HmMatch, passing $n, n^{\prime}, L H$, and $S$ as parameters. HmMatch identifies differences and correspondences between nodes in $G$ and $G^{\prime}$ (line 9), and creates and returns $N$, the set of matched nodes and corresponding labels ("modified" or "unchanged"). Finally, CalcDiff returns $N, C, I$, and $M$ (line 12).

In the next section, we discuss the ECFG, the representation we use to perform node matching. Then, we discuss hammocks and how we process them. Finally, we present and explain our hammock-matching algorithm, HmMatch.

\subsubsection{Enhanced Control-Flow Graphs}

When comparing two methods $m$ and $m^{\prime}$, the goal of our algorithm is to find, for each statement in $m$, a matching (or corresponding) statement in $m^{\prime}$, based on the method structure. Thus, the algorithm requires a modeling of the two methods that (1) explicitly represents their structure, and (2) contains sufficient information to identify differences and similarities between them. Although CFGs can be used to represent the control structure of methods, traditional CFGs do not suitably model many object-oriented constructs. To suitably represent object-oriented constructs, and model their behavior, we define the ECFG. ECFGs extend traditional CFGs and are tailored to represent objectoriented programs. In the following, we illustrate how the ECFG represents some important Java features.

\section{Dynamic Binding}

Because of dynamic binding, an apparently harmless modification of a program may affect call statements in a different part of the program with respect to the change point. For example, class-hierarchy changes may affect calls to methods in any classes in the hierarchy, and adding a method to a class may affect calls to the methods with the same signature in its superclasses and subclasses.

We illustrate how we model a call site, in which a method $m$ is called on an object $o$, to capture these modifications. First, we create a call and a return node. Then, for each dynamic type $T$ that can be associated with $o$, we create a callee node. A callee node represents the method that is bound to the call when the type of $o$ is $T$, and is labeled with the signature of that method. We also create (1) a call edge from the call node to each callee node, labeled with the type that causes such a binding, and (2) a return edge from each callee node to the return node. Note that if the call is static (i.e., not virtual), there is only one callee node.

To illustrate, consider method D.m3 in $P$ (Figure 1). The ECFG for D.m3 (Figure 3(a)), contains two callee nodes (3 

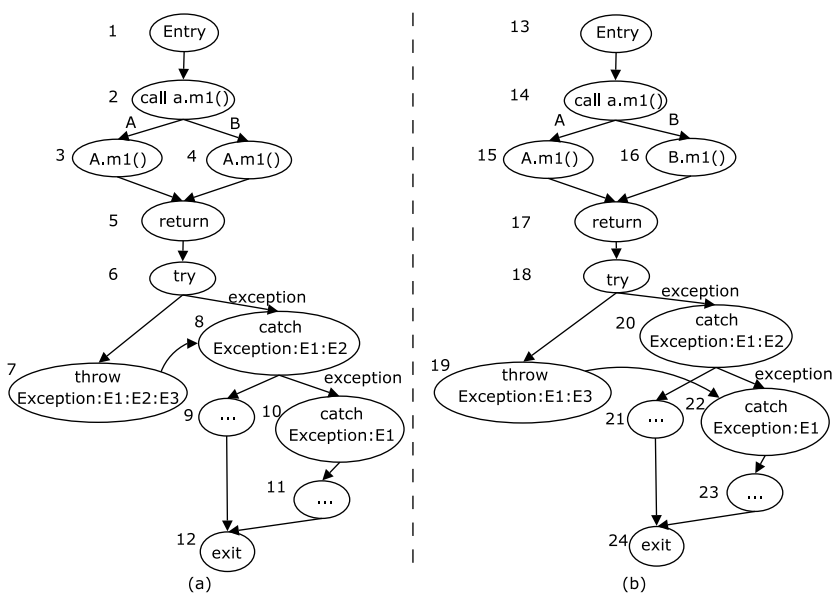

Figure 3. ECFGs for D.m3 in $P$ and $P^{\prime}$ (Figure 1).

and 4) because $a$ 's dynamic type can be either $A$ or $B$. Both added nodes correspond to the same method, and thus have the same label, because $B$ does not override method $m 1$.

Consider now one of the two differences between $P$ and $P^{\prime}$ in Figure 1: the redefinition of method $m 1$ in $B$. Such a change causes a possibly different behavior in $P$ and $P^{\prime}$ for the call to $a . m 1$ in method D.m3: if the dynamic type of $a$ is $B$, the call results in an invocation of method $A . m 1$ in $P$ and results in an invocation of method B. $m 1$ in $P^{\prime}$.

Figure 3(b) shows how the different binding, and the possibly different behavior, is reflected in the ECFG for method D. $m 3$ : the call edge labeled $B$ from the call node for a. $m 1$ (i.e., the call edge representing the binding when $a$ 's type is $B$ ) is now connected to a new callee node that represents method B.m1. This difference between the ECFGs for D.m3 in $P$ and $P^{\prime}$ lets our analysis determine that this call to a.m1 may behave differently in $P$ and $P^{\prime}$. Note that a simple textual comparison would identify the addition of the method, but it would require a manual inspection of the code (or some further analysis) to identify the points in the code where such change can affect the program's behavior.

\section{Variable and object types}

When modifying a program, changing the type of a variable may lead to changes in program behavior (e.g., changing a long to an int). To identify these kinds of changes, in our representation, we augment the name of scalar variables with type information. For example, we identify a variable $a$ of type double as a_double. This method for representing scalar variables reflects any change in the variable type in the locations where that variable is referenced.

Another change that may lead to subtle changes in program behavior is the modification of class hierarchies (e.g., moving a class from one hierarchy to another, by changing the class that it extends). Effects of these changes that result in different bindings in $P$ and $P^{\prime}$ are captured by our method-call representation. Other effects, however, must be specifically addressed. To this end, instead of explicitly representing class hierarchies, we encode the hierarchy information at points where a class is used as an argument to operator instanceof, as an argument to operator cast, as a type of a newly created exception, and as the declared type of a catch block. To encode the type information, we use globally-qualified class names. A globallyqualified class name for a class contains the entire inheritance chain from the root of the inheritance tree (i.e., from class java.lang.Object) to its actual type. ${ }^{2}$ The interfaces that are implemented by the class are also included in globally-qualified names. If a class implements more than one interface, the names of the interfaces are inserted in alphabetical order. This method reflects changes in class hierarchies in the locations where the change may affect the program behavior. For example, nodes 7 and 19 in Figure 3 show the globally-qualified name for class $E 3$ in $P$ and $P^{\prime}$, respectively.

\section{Exception Handling}

As for dynamic binding, program modifications in the presence of exception-handling constructs can cause subtle side effects in parts of the code that have no obvious relation to the modifications. For example, a modification of an exception type or a catch block can cause a previously caught exception to go uncaught in the modified program, thus changing the flow of control in unforeseen ways.

To identify these changes in the program, we explicitly model, in the ECFG, exception-handling constructs in Java code. We represent such constructs using an approach similar to that used in Reference [3]. For each try statement, we create a try node and an edge between the try node and the node that represents the first statement in the try block.

We then create a catch node and a CFG to represent each catch block of the try statement. Each catch node is labeled with the type of the exception that is caught by the corresponding catch block. An edge connects the catch node to the entry of the CFG for the catch block.

An edge, labeled "exception", connects the try node to the catch node for the first catch block of the try statement. That edge represents all control paths from the entry node of the try block along which an exception can be propagated to the try statement. An edge labeled "exception" connects also the catch node for a catch block $b_{i}$ to the catch node for catch block $b_{i+1}$ that follows $b_{i}$ (if any). This edge represents all control paths from the entry node of the try block along which an exception is (1) raised, (2) propagated to the try statement, and (3) not handled by any of the catch blocks that precede $b_{i+1}$ in the try statement.

\footnotetext{
${ }^{2}$ For efficiency, we exclude class Object from the name, except that for class Object itself.
} 
Our representation models finally blocks by creating a CFG for each finally block, delimited by finally entry and $f$ nally exit nodes. An edge connects the last node in the corresponding try block to the finally entry node. The representation also contains one edge from the last node of each catch block related to the finally to the finally entry node. If there are exceptions that cannot be caught by any catch block of the try statement and there is at least one catch block, an edge connects the catch node for the last catch block to the finally entry node.

Because the information we use to build the exceptionrelated part of the ECFG is computed through interprocedural exception analysis [14], we can represent both intra- and inter-procedural exception flow. If an exception is thrown in a try block for a method $m$, the node that represents the throw statement is connected to (1) the catch block in $m$ that would catch the exception, if such a catch block exists, (2) the finally entry node, if no catch block can catch the exception and there is a finally block for the considered try block, or (3) to the exit node of $m$ 's ECFG, otherwise. Conversely, if an exception is thrown in method $m$ from outside a try block, the node that represents the throw statement is always connected to the exit node of $m$ 's ECFG.

For example, consider again method D.m3 in $P$ (Figure 1) and its ECFG (Figure 3(a)). The ECFG contains a try node for the try block (node 6) and catch nodes for the two catch blocks associated with the try block (nodes 8 and 10). The catch nodes are connected to the entry nodes of the CFGs that represent the corresponding catch blocks (nodes 9 and 11). Also, the node that represents the throw statement in the code is connected to the catch node whose type matches the type of the exception (edge $\langle 7,8\rangle$ ).

Consider now the other difference between $P$ and $P^{\prime}$ : the modification in the type hierarchy that involves class $E 3$. $E 3$ is a subclass of $E 2$ in $P$ and a subclass of $E 1$ in $P^{\prime}$. Such a change causes a possibly different behavior in $P$ and $P^{\prime}$ because the exception thrown in method D.m3 is caught by different catch blocks in $P$ and $P^{\prime}$. Because, in $P^{\prime}$, class $E 3$ is no longer a subclass of $E 2$, edge $\langle 7,8\rangle$, which connects the throw node to the catch node for exception $E 2$ in $P$, is replaced by edge $\langle 19,22\rangle$, which connects the throw node to the catch node for exception $E 1$ in $P^{\prime}$.

Figure 3(b) shows how the possibly different behavior is reflected in our representation: the node that represents the throw statement is connected to two different catch nodes in the ECFGs for D.m3 in $P$ and $P^{\prime}$. In addition, the nodes that represent the throw statement in $P$ and $P^{\prime}$ (nodes 7 and 19) differ because of the use of globally-qualified names for the exception types. These differences between the two ECFGs let our analysis determine that, if the throw statement is traversed, $P$ and $P^{\prime}$ may behave differently. Note that this would occur also for an exception that propagates outside the method, due to the difference between the globally-qualified names in nodes 7 and 19. Again, a simple textual comparison would let us identify only the change in the type of $E 3$, whereas identifying the side effects of such a change would require further analysis.

\section{Synchronization}

Java provides explicit support for threading and concurrency through the synchronized construct. Using such construct, Java programmers can enforce mutual exclusion semaphores (mutexes) or define critical sections, that is, atomic blocks of code. Synchronized areas of code can be declared at the block, method, and class level.

In the ECFG, we account for synchronized areas of code by creating two special nodes: synchronize start and synchronize end. A synchronize start node is added before the node that represents the first statement of a synchronized area of code. Analogously, a synchronize end node is added after the node that represents the last statement of a synchronized area of code.

In a program that uses synchronized constructs, changes in behavior can occur because (1) an area of code that was not synchronized becomes synchronized, (2) an area of code that was synchronized is no longer synchronized, or (3) a synchronized area is expanded or contracted. In the ECFG, these cases are suitably captured by addition, removal, or replacement of synchronize start and synchronize end nodes.

\section{Reflection}

In Java, reflection provides runtime access to information about classes' fields and methods, and allows for using such fields and methods to operate on objects. In the presence of reflection, our representation can fail to capture some of the behaviors of the program. Although some uses of reflections can be handled through analysis, others require additional, user-provided information. In our work, we assume that such information is available and can be leveraged for the analysis. In particular, for dynamic class loading, we assume that the classes that can be loaded (and instantiated) by name at a specific program point are specified by the user.

\subsubsection{Hammocks}

Our algorithm uses hammocks and hammock graphs for its comparison of two methods. Hammocks are single-entry, single-exit subgraphs [2] and provide a way to impose a hierarchical structure on the ECFGs that facilitates the matching. Formally, if $G$ is a graph, a hammock $H$ is an induced subgraph of $G$ with a distinguished node $V$ in $H$ called the entry node and a distinguished node $W$ not in $H$ called the exit node such that

1. All edges from $(G-H)$ to $H$ go to $V$.

2. All edges from $H$ to $(G-H)$ go to $W$. 


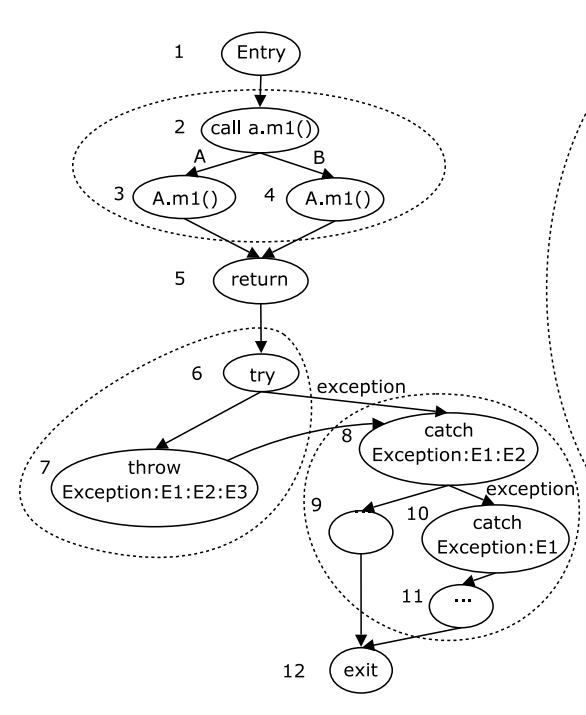

(a)

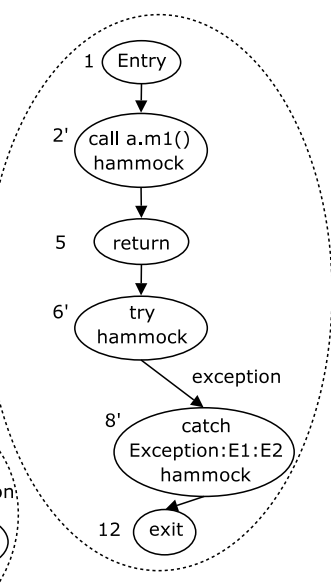

(b)

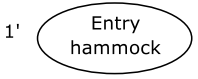

(c)
Figure 4. ECFG for D.m3 (a), intermediate hammock graph for D.m3 (b), and resulting hammock node for D.m3 (c)

Similar to the approach used in Reference [7], once a hammock is identified, our algorithm reduces it to a hammock node in three steps. First, the set of nodes in the hammock is replaced by a new node. Second, all incoming edges to the hammock are redirected to the new node. Third, all edges leaving the hammock are replaced by an edge from the new node to the hammock exit. The resulting graph at each intermediate step is called a hammock graph.

Figure 4 illustrates the steps and hammock graphs in reducing ECFGs for $P$ to a single node. The regions inside the dotted lines-nodes 2-4, nodes 6 and 7, and nodes 8 11 -in Figure 4(a) represent the three hammocks that are identified and then replaced by hammock nodes 2', 6' and 8', respectively, as shown in Figure 4(b). Then, all nodes in Figure 4(b) are identified as a hammock and reduced to a single node, as shown in Figure 4(c). To identify all hammocks, we use an algorithm described in Reference [2].

A hammock $H$ with start node $s$ is minimal if there is no hammock $H^{\prime}$ that (1) has the same start node $s$, and (2) contains a smaller number of nodes. Hereafter, when we use the term hammock, we always refer to a minimal hammock, unless otherwise stated.

\subsubsection{Hammock Matching Algorithm}

Our hammock matching algorithm, HmMatch, is given in Figure 5. The algorithm is based on Laski and Szermer's algorithm for finding an isomorphism between two graphs [7]. HmMatch takes as input $n$ and $n^{\prime}$, two hammock nodes, $L H$, an integer indicating the maximum lookahead, and $S$, a threshold for deciding whether two hammocks are similar procedure HmMatch

Input: hammock node in original version $n$, hammock node in modified version $n^{\prime}$ maximum lookahead $L H$ hammock similarity threshold $S$

Output: set of pair $\langle\{$ node,node $\}$, label $\rangle N$

Use: $\operatorname{succs}(A)$ returns set of successors of each node $a$ in $A$ $\operatorname{comp}(m, n, S, N)$ returns true if $\mathrm{m}$ and $\mathrm{n}$ are matched

edgeMatching $\left(n, n^{\prime}\right)$ returns matched outgoing edge pairs

Declare: stack of $\langle$ node, node $\rangle S T$ current depth $d$ expanded graphs $G_{e}$ and $G_{e}^{\prime}$ current nodes $c$ and $c^{\prime}$ lookahead node sets $L$ and $L^{\prime}$ pair $\langle$ node, node $\rangle$ match

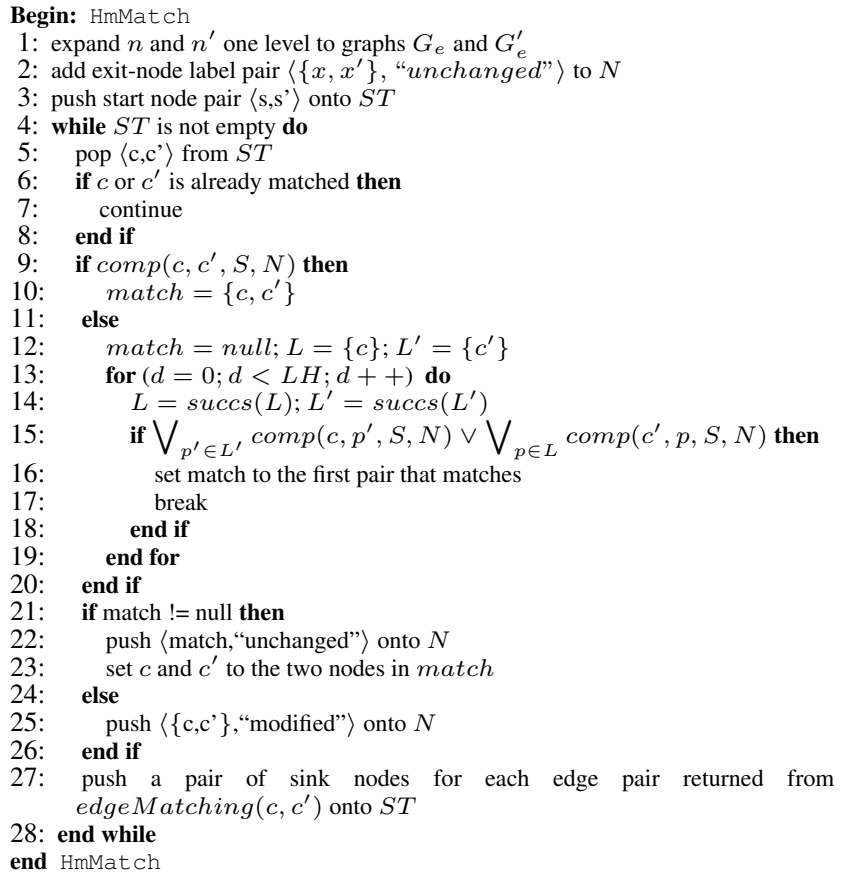

Figure 5. A hammock matching algorithm

enough to be considered a match. The algorithm outputs $N$, a set of pairs whose first element is, in turn, a pair of matching nodes, and whose second element is a label that indicates whether the two nodes are "unchanged" or "modified."

To increase the number of matches, we modified Laski and Szermer's algorithm to allow for the matching of hammocks at different nesting levels. This modification accounts for some common changes that we encountered in our preliminary studies, such as the addition of a loop or of a conditional statement at the beginning of a code segment. In the following, we first describe algorithm HmMatch and then present an example of use of the algorithm on the code in Figure 1.

HmMat $c h$ expands the two input hammock nodes, $n$ and $n^{\prime}$, into two hammock graphs, $G_{e}$ and $G_{e}^{\prime}$ (line 1$)$. In the expansion process, a dummy exit node is added and all edges from nodes in the hammock to the actual exit node are redirected to the dummy node. At line 2, HmMatch adds the two dummy exit nodes as a pair of matching nodes to set $N$. Then, the algorithm starts matching nodes in the two 
graphs by performing a depth-first pairwise traversal of $G_{e}$ and $G_{e}^{\prime}$, starting from their start nodes. Thus, at line 3, the pair of start nodes is added to stack $S T$, which functions as a worklist. Each iteration of the main while loop (lines 4-28) extracts one node pair from the stack and checks whether the two nodes match. The body of the loop first checks whether any node in the current pair is already matched (line 6). A matched node that has already been visited must not be considered again; in this case, the algorithm continues by considering the next pair in the worklist (line 7).

To compare two nodes, HmMatch invokes $\operatorname{comp}\left(c, c^{\prime}, S, N\right)$ (line 9), where $c$ and $c^{\prime}$ are the two nodes to compare, $S$ is the similarity threshold for matching hammocks, and $N$ is the set of matching nodes. Unless $c$ and $c^{\prime}$ are hammocks, comp returns true if the two nodes' labels are the same. If $c$ and $c^{\prime}$ are hammocks, comp (1) recursively calls HmMatch to obtain the set of matched and modified pairs, and (2) computes the ratio of unchange-matched pairs in the set to the number of nodes in the smaller hammock. If the ratio is greater than threshold $S$, comp returns true (i.e., the two hammocks are matched) and pushes all pairs in the set returned by Hmatch onto $N$. Otherwise, comp returns false.

If two nodes $c$ and $c^{\prime}$ are matched (i.e., comp returns true), they are stored in variable match as a pair (line 10) and further added to the set of matched nodes with label "unchanged" (line 22). Otherwise, HmMatch tries to find a match for $c$ (resp., $c^{\prime}$ ) by examining $c^{\prime}$ 's (resp., $c$ 's) descendants up to a given maximum lookahead (lines 12-19). First, match is initialized to null, and the lookahead sets $L$ and $L^{\prime}$ are initialized to contain only the current nodes (line 12). The algorithm then executes the for loop until a match is found or depth $d$ reaches the maximum lookahead $L H$ (lines 13-19). At each iteration, the algorithm updates $L$ and $L^{\prime}$ to the sets of successors of their members, obtained by calling procedure succs (line 14). succs $(L)$ returns, for each node $l$ in $L$ and each outgoing edge from $l$, the sink of such edge. If node $l$ is a hammock node, succs returns a set that consists of the start node and the exit node of the hammock. In this way, a match can occur between nodes in hammocks at different nesting levels. After computing the lookahead sets $L$ and $L^{\prime}$, the algorithm compares each node in set $L^{\prime}$ with $c$ and each node in set $L$ with $c^{\prime}$ (line 15). If there is a match, the search stops, and the first matching pair found is stored in variable match (lines 16-17). The matching pair is then added to the set of matched nodes with label "unchanged" (line 22). After two nodes have been matched as unchanged, $c$ and $c^{\prime}$ are set to be the two nodes in the matching pair (line 23).

If no matching is found, even after the lookahead, $c$ and $c^{\prime}$ are added to the set of matched nodes with label "modified."

After processing nodes $c$ and $c^{\prime}$, the outgoing edges from the two nodes are matched by calling edgeMatching $\left(c, c^{\prime}\right)$.
edgeMatching matches outgoing edges from $c$ and $c^{\prime}$ based on their labels. For each pair of matching edges, the corresponding sink nodes are pushed onto worklist $S T$ (line 27). At this point, the algorithm continues iterating over the main while loop until $S T$ is empty.

When the algorithm terminates, all nodes in the old version that are not in any pair (i.e., that have not been matched to any other node) are considered deleted nodes. Similarly, all nodes in the new version that are not in any pair are considered added nodes.

To better illustrate HmMatch, we consider a partial run of CalcDiff on the example code in Figure 1. In particular, we consider the execution from the point at which the pair of methods D.m3 in $P$ and $P^{\prime}$ is compared (line 5). At line 6 of CalcDiff, the ECFGs for the methods are created, and at lines 7 and 8 of the algorithm, hammocks in the ECFGs are identified and reduced to single hammock nodes. Then, at line 9, CalcDiff calls HmMatch, passing it the two hammock nodes. For the example, we assume that the lookahead threshold $(L H)$ is 1 , and that the hammock similarity threshold $(S)$ is 0.5 .

HmMatch first expands the hammock nodes, adds the dummy exit nodes, and suitably connects them (line 1). Fig. 6 shows the resulting hammock graphs for the original and modified version of the program. Then, HmMat ch adds dummy exit nodes 7 and 13 as a pair of matching nodes to set $N$ (line 2) and adds the pair of start nodes $\langle 1,8\rangle$ to stack $S T$ (line 3).

In the first iteration over the main while loop, the algorithm extracts node pair $\langle 1,8\rangle$ from $S T$ (line 5). Because neither node is already matched, the algorithm compares the two nodes by calling $\operatorname{comp}(1,8,0.5, N)$ (line 9), which compares the nodes' labels and returns true. Therefore, the algorithm sets match to this pair (line 10), adds the pair of nodes to $N$ with label "unchanged," and sets $c$ and $c^{\prime}$ to be nodes 1 and 8 (lines 22-23), which in this case leaves $c$ and $c^{\prime}$ unchanged. At this point, the outgoing edges from 1 and 8 are matched by calling edgeMatching $(1,8)$. Each node in the entry pair has only one outgoing edge, and the two edges match, so the pair of sink nodes $\langle 2,9\rangle$ is pushed onto the worklist.

In the second iteration over the main while loop, because nodes 2 and 9 are not already matched and are both hammock nodes, comp (line 9) calls HmMatch $(2,9,1,0.5)$. HmMatch expands nodes 2 and 9 to get the two graphs shown in Fig. 7, matches the dummy exit nodes 17 and 21 , and pushes the pair of start nodes $\langle 14,18\rangle$ onto $S T_{1}{ }^{3}$ This pair is then extracted from the stack and compared (lines 5-9). Because both nodes have the same label, they are matched, and the pair is pushed onto $N_{1}$ with label "unchanged" (lines 22-23). edgeMatching is then called

\footnotetext{
${ }^{3}$ We use the subscript notation to distinguish variables in recursively called procedures.
} 


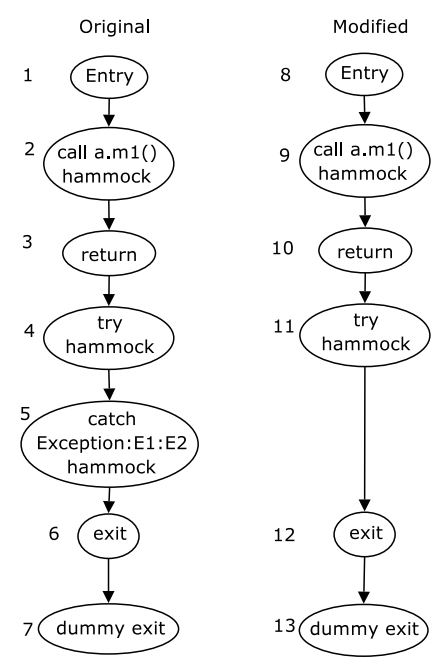

Figure 6. Hammock graphs for the original and modified version of D.m3.

on the two nodes in the pair, 14 and 18; edgeMatching matches like-labeled edges and the two pairs of sink nodes $\langle 15,19\rangle$ and $\langle 16,20\rangle$ are pushed onto $S T_{1}$.

In the next iteration over the main loop, the nodes in pair $\langle 15,19\rangle$ are compared, matched, and pushed onto $N_{1}$ with label "unchanged," and the pair of direct successors of nodes 15 and $19,\langle 17,21\rangle$, is then pushed onto $S T_{1}$. At the following loop iteration, the nodes in pair $\langle 16,20\rangle$ are compared. Because they do not match, a lookahead is performed, trying to match nodes 16 and 21 and nodes 20 and 17. Because neither comparison is successful, the pair $\langle 16,20\rangle$ is added to $N_{1}$ with label "modified," and the pair of successors $\langle 17,21\rangle$, is then pushed onto $S T_{1}$ (so the pair appears twice on the stack). In the next two iterations, the pair $\langle 17,21\rangle$ is processed: because both nodes are already matched (being the dummy exits), the algorithm skips them. At this point, $S T_{1}$ is empty and HmMatch returns to the calling procedure, comp. Because 3 out of 4 nodes are unchangematched, and the similarity threshold is 0.5 , comp classifies the two hammocks as matched. Therefore, the pairs in $N_{1}$ are added to $N$, comp returns true (line 9), pair $\langle 2,9\rangle$ is pushed onto $N$ with label "unchanged" (line 22), and pair $\langle 3,10\rangle$ is pushed onto $S T$ (line 27).

Pair $\langle 3,10\rangle$ is then matched and pair $\langle 4,11\rangle$ is pushed onto $S T$ and then compared in the next iteration. For the sake of space, we do not show the comparison of these two hammocks and the rest of the execution of the algorithm. The part of the example shown so far already illustrates the main parts of the algorithm, including the matching of hammock nodes and the lookahead.

\subsection{Worst-Case Time Complexity}

The dominating cost of CalcDiff is the matching at the node level. Let $m$ and $n$ be the number of nodes in all
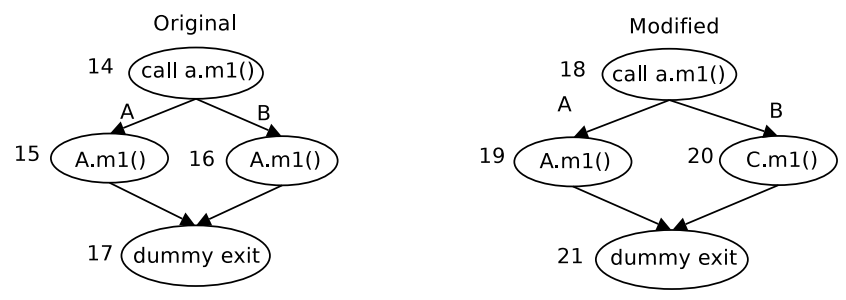

Figure 7. Hammock graphs for hammock nodes 2 and 9 in Fiugure 6.

matched methods in the original and modified versions of a program. Let $k$ be the maximum number of nodes in a method, and let the maximum lookahead be greater than $k$. In the worst case, if no matching of hammocks at different nesting levels occurs, the algorithm compares each node in a method with all nodes in the matching method (at most, $k$ ), leading to a worst-case complexity of $O(k \cdot \min (m, n))$. If matching of hammocks at different nesting levels occurs, the algorithm may compare a pair of nodes more than once. To decide whether two hammocks are matched, HmMatch compares each node in one hammock with nodes in the other hammock and counts the number of matched nodes. If lookahead is performed, the same pairs of nodes are compared again in the context of the new pair of hammocks. The number of times the same pairs of nodes are compared depends on the maximum nesting depth of hammocks and on the maximum lookahead. If $d$ is the maximum nesting level of hammocks and $l$ is the maximum lookahead, the worst-case complexity of our algorithm is then $O(k \cdot \min (d, l) \cdot \min (m, n))$.

\section{Studies}

To evaluate our algorithm, we implemented a tool, JDIFF, and performed three studies on a real, medium-sized program. In this section, we describe our experimental setup, present the studies, and discuss their results.

\subsection{Experimental Setup}

JDIFF is a Java tool that implements our differencing algorithm. The tool consists of two main components: a differencing tool and a graph-building tool. The differencing tool inputs the original and modified versions of a program, compares them, and outputs sets of pairs of matching classes, methods, and nodes. To build ECFGs, the tool leverages the capabilities of JABA (Java Architecture for Bytecode Analysis), ${ }^{4}$ a Java-analysis front-end developed within our research group. We are currently using JDIFF in our JABA-based impact analysis and regression testing tools [10].

As a subject for our studies, we use JABA, the analysis tool described above. JABA contains 550 classes, 2800

\footnotetext{
${ }^{4}$ http://www.cc.gatech.edu/aristotle/Tools/jaba. html
} 
methods, and approximately 60KLOC. To evaluate the effectiveness of our algorithm, we ran JDIFF on pairs of successive versions of JABA extracted from its CVS repository.

For the timing experiments, we used two dedicated Pentium4 $2.80 \mathrm{GHz}$ PCs, with $1 \mathrm{~GB}$ of memory, running GNU/Linux 2.4.22.

There are several threats to the validity of our studies. An external threat exists because we conducted the studies using only one subject program. Thus, we cannot claim generality for our results. However, the subject program is a real program, that is used constantly by students and researchers in and outside our group, and the changes considered are real changes that include bug fixes and feature enhancements. Another external threat to validity exists for the first study: we used only one test suite throughout the study. Different test suites may generate different results.

Threats to internal validity mostly concern possible errors in our algorithm implementations and measurement tools that could affect outcomes. To control for these threats, we validated the implementations on known examples and performed several sanity checks.

\subsection{Study 1}

The goal of Study 1 is to assess the effectiveness of our algorithm for a particular task-coverage estimation.

For the discussion, let $P$ represent the original version of the program, $P^{\prime}$ represent the modified version of $P$, and $T$ represent a test suite used for testing $P$. The coverage information for $P, C_{P}$, records, for each test case $t \in T$, entities exercised by $t$. Coverage estimation estimates $C_{P^{\prime}}$ from $C_{P}$ and the correspondences between entities in $P$ and $P^{\prime}$. In this study, we consider a node in the ECFG as an entity in the program. Therefore, the coverage information is expressed as a set of nodes that each test case $t$ exercises.

We used four versions of JABA and its development test suite $T$, which contains 707 test cases and covers approximately $60 \%$ of the program. The size of the change sets between versions in the pairs ranges from a few modified methods to approximately 20 methods.

Let $P_{i}$ represent version $i$ of the JABA program, where $1 \leq i \leq 4$. We first ran the test suite $T$ on each version $P_{i}$ to collect $C_{P_{i}}$. Second, for each $\left\langle P_{1}, P_{i}\right\rangle$ pair, where $2 \leq i \leq 4$, we computed the node correspondences between $P_{1}$ and $P_{i}$ using JDIFF. Third, we computed the estimated coverage as follows:

- For each node $n$ in the modified version (i.e., the second element of each $\left\langle P_{1}, P_{i}\right\rangle$ pair), if there exists a correspondence between node $n$ and some node $n^{\prime}$ in the original version (i.e., $P_{1}$ ), then we estimate that $n$ is exercised by the test cases in $T$ that exercised $n^{\prime}$.

- If there is no such correspondence, we visit the predecessors of $n$ in the ECFG until either the predecessor is a branch or the predecessor is a node $q$ that has a correspondent node $q^{\prime}$ in $P_{1}$. In the former case, $n$ is designated as "not exercised." In the latter case, we estimate that $n$ is exercised by all the test cases in $T$ that exercised $q^{\prime}$ (because $n$ is in the same control-dependence region of $q$ ).

Finally, we compared the estimated and the actual coverage, and recorded the number of nodes whose coverage is correctly estimated.

\begin{tabular}{|l|c|c|c|c|}
\hline Pair & $\begin{array}{c}\text { Number } \\
\text { of Nodes }\end{array}$ & $\begin{array}{c}\text { Avg. } \\
\text { Covered }\end{array}$ & $\begin{array}{c}\text { Avg. Correct// } \\
\text { Test Case }\end{array}$ & $\begin{array}{c}\text { Correct/ } \\
\text { Test Suite }\end{array}$ \\
\hline v1,v2 & 22026 & 12591.75 & 98.57 & 97.17 \\
v1,v3 & 22063 & 12620.75 & 98.46 & 97.17 \\
v1,v4 & 22315 & 12661.23 & 98.03 & 96.20 \\
\hline
\end{tabular}

Table 1. Coverage-estimation results for part 1 of Study 1.

Table 1 shows, for each pair of versions, the number of nodes in the parts of the modified version that we consider (Number of Nodes), the average number of nodes covered by a test case (Avg. Covered), the number of nodes whose coverage of each test case $t \in T$ is correctly estimated, averaged over all test cases (Avg. Correct / Test Case), and the number of nodes whose coverage of the entire test suite is correctly estimated (Correct / Test Suite). For example, $v 3$ contains 22,063 nodes, and a single test case exercised about 12,620 nodes on average. For this set of nodes, on average, $98.46 \%$ of the nodes' coverage for a single test case and $97.17 \%$ of the nodes' coverage for the entire test suite are correctly estimated. The results show that estimated coverage is high for the pairs we studied but that, as expected, it slightly degrades as the change set becomes larger (i.e., when considering pairs $\langle v 1, v 3\rangle$ and $\langle v 1, v 4\rangle$ ).

To further evaluate the effectiveness of our algorithm when used for coverage estimation, we applied it to program versions with a large number of changes. To this end, we extracted four additional versions of JABA from its CVS by selecting a period of time in which JABA was undergoing a major restructuring. We call these versions $v a, \ldots, v d$. We constructed pairs of versions using the first version $v a$ as the first element of each pair. The sizes of the change sets in these pairs are 15 methods for $\langle v a, v b\rangle, 100$ methods for $\langle v a, v c\rangle$, and 150 methods for $\langle v a, v d\rangle$. The test suite for these versions consists of 155 test cases, which covers approximately $45 \%$ of the program.

Table 2 gives the data for this set of pairs of versions in the same format as Table 1. The results show that, also for this set of versions, the quality of estimated coverage degrades when the change sets becomes larger. However, for the largest change set, our algorithm still computes estimated coverage that correctly matches the actual coverage for $84.70 \%$ of the nodes (on average) for the single test 


\begin{tabular}{|c|c|c|c|c|}
\hline Pair & $\begin{array}{c}\text { Number } \\
\text { of Nodes }\end{array}$ & $\begin{array}{c}\text { Avg. } \\
\text { Covered }\end{array}$ & $\begin{array}{c}\text { Avg. Correct/ } \\
\text { Test Case }\end{array}$ & $\begin{array}{c}\text { Correct/ } \\
\text { Test Suite }\end{array}$ \\
\hline va,vb & 19639 & 12199.60 & 96.25 & 95.30 \\
va,vc & 20076 & 12343.54 & 86.08 & 80.97 \\
va,vd & 20258 & 12316.49 & 84.70 & 77.81 \\
\hline
\end{tabular}

Table 2. Coverage-estimation results for part 2 of Study 1.

cases, and for $77.81 \%$ of the nodes for the entire test suite. Our results are consistent with the findings in Reference [1].

\subsection{Study 2}

The goal of Study 2 is to measure the efficiency of JDIFF for various values of lookahead, $L H$, and hammock similarity threshold, $S$.

In this study, we used the first three pairs of versions of JABA that we used in Study 1. We ran JDIFF on each pair of versions $\left\langle P_{1}, P_{i}\right\rangle$, where $2 \leq i \leq 4$, with different values for $L H$ and $S$, and collected the running times. This running time includes the time for creating the ECFGs, as well as comparing and matching classes, interfaces, methods, and nodes in the two programs.

Figure 8 shows the running time (in seconds) of JDIFF on a number of pairs of versions, lookaheads, and similarity thresholds. The x-axis represents the value of $L H$, and the y-axis represents the running time for each pair of versions and for two different values of $S$. For example, JDIFF took about 375 seconds when run on versions $\langle v 1, v 2\rangle$ for $(S>0)$ and $L H$ of 10 . We present the results for only two values of $S(S=0$ and $S>0$ ) because we found that, for this subject, the running time is almost the same for $S=0.2,0.4,0.6$, and 0.8. Our results show that, when $L H$ is constant, the value of $S$ affects the running time of the comparison. Intuitively, with $S=0$, the algorithm matches a hammock in the original program's ECFG with the first hammock found in the modified version's ECFG. Thus, each hammock is compared at most once, and the running time is almost the same regardless of the value of $L H$. In addition, because each hammock is compared at most once, the running time for these cases is less than for cases where $S>0$, where a hammock may be compared more than once. For $S>0$, the number of times a hammock is compared depends on the lookahead and on the actual changes. As shown in the results, only in this case does the time slightly increase when the lookahead increases. Note that, in all cases, JDIFF took less than seven minutes to compute the differences between a pair of versions.

\subsection{Study 3}

The goal of Study 3 is to evaluate the effectiveness of our algorithm compared to Laski and Szermer's algorithm [7].

To do this, we compared the number of nodes that each algorithm matches. For the study, we implemented Laski

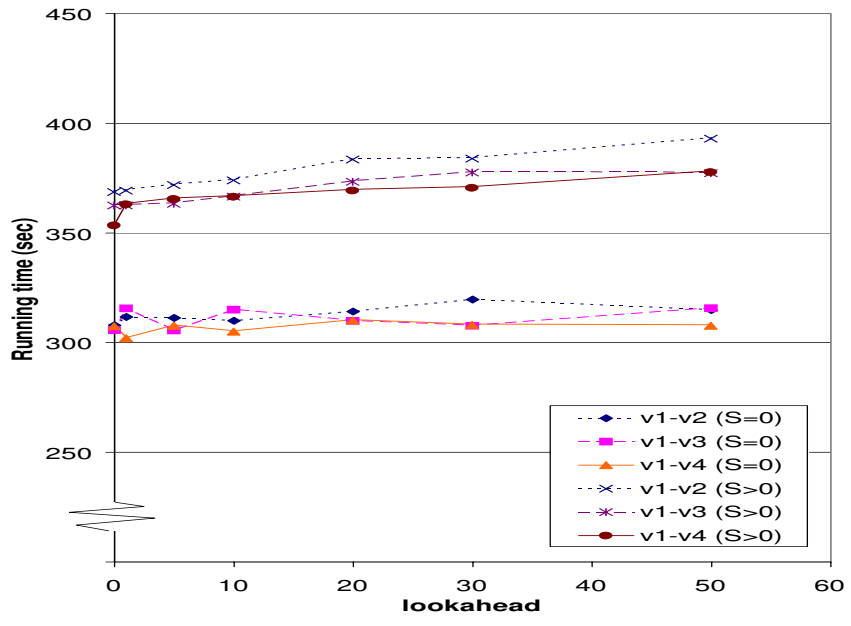

Figure 8. Average time (sec) for various pairs of versions, lookaheads, and similarity thresholds.

and Szermer's algorithm (LS) by modifying our tool. Reference [7] does not discuss how LS handles some specific cases. For example, when two hammocks have the same label, they are expanded and compared, but the algorithm behavior is undefined in the case in which the expanded graphs cannot be made isomorphic by applying node renaming, node removing, and node collapsing. We handle those cases in the same way for both algorithms. There are three differences between the two algorithms: (1) LS does not use the lookahead but searches the graphs until the hammock exit node is found; (2) LS does not allow the matching of hammocks at different nesting levels; and (3) LS does not use the hammock similarity threshold but decides whether two hammocks are matched by comparing the hammocks' entry nodes only.

We ran both algorithms on the first three pairs of versions of JABA used in Study 1, and counted the number of nodes in each group of added, deleted, modified, and unchanged nodes. We consider only nodes in modified methods because added, deleted, and unchanged methods do not show differences in matching capability between the two algorithms. In our preliminary studies, we found that LS identified, in the modified methods, about 5,000 nodes as added and about 5,000 nodes as deleted.

Figure 9 presents the results of this study. The horizontal axis represents the size of $L H$, and the vertical axis shows, for each pair of versions and values of $S$, the percent increase in the number of matched nodes over the number of nodes identified as added by LS. For example, our algorithm (for $S>0$, and $L H=20$ ) matches about $55 \%$ more nodes than LS when both algorithms run on the same pair $\langle v 1, v 2\rangle$. In this case, our algorithm matches 2750 additional nodes over the number of nodes matched by LS. The results show that the increase achieved by our algorithm ranges from about $17 \%$ to over $65 \%$. Note that added nodes 


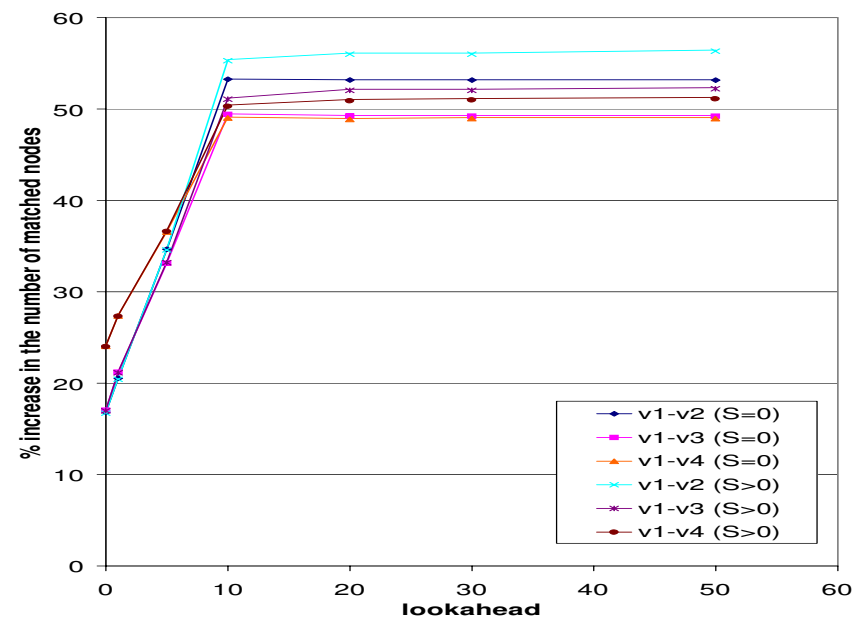

Figure 9. Percentage increase in matched nodes.

identified by LS or CalcDiff can be classified as (1) code that is actually added, or (2) code that cannot be matched because of the limitations of the algorithms. Therefore, to measure the relative effectiveness of the two algorithms, the percentage should be computed using the number of nodes only in the second category. In this sense, the percent improvement that we measured in our study is an underestimate of the actual improvement.

The results also show that the number of matched nodes increases when $L H$ increases, which is intuitively reasonable. Finally, the results show that the number of matched nodes is slightly higher in the case of $S>0$ than in the case of $S=0$, for $L H>10$.

\section{Related Work}

There are a number of existing techniques for computing differences between two versions of a program that are related to ours. The UNIX diff utility [9], as discussed in the Introduction, compares two text files line-by-line and outputs differences in the files. However, because diff compares files line-by-line, it does not recognize differences that can occur because of changes in object-oriented features such as changes related to polymorphism and exception handling. In contrast, our differencing technique does handle these object-oriented features, and thus, can provide information that can be used for various software engineering tasks.

BMAT [15] (binary matching tool) is similar to our algorithm. However, it performs matching on both code and data blocks between two versions of a program in binary format. BMAT uses a number of heuristics to find matches for as many blocks as possible. Being designed for the purpose of program profile estimation, BMAT does not provide information about differences between matched entities (unlike our algorithm). Moreover, BMAT does not compute information about changes related to object-oriented constructs.

Semantic diff [6], compares two versions of a program procedure-by-procedure. Semantic diff computes a set of input-output dependencies for each procedure and identifies the differences between two sets from the same procedure in the original and the modified versions. However, semantic diff is performed only at the procedure level and may miss some changes that do not affect the dependencies of variables (e.g., changing branch conditions) but may drastically change the behavior of the program. Furthermore, because there is no input-output dependency changes, it will fail to detect some kinds of changes (e.g., constant value changed) that may affect the program behavior. Conversely, our technique is able to identify these differences.

Horwitz's approach [4] computes both syntactic and semantic differences between two programs using a partitioning algorithm. Horwitz's technique is based on the program representation graph (PRG). Because PRGs are defined only for programs written in a language with scalar variables, assignment statements, conditional statements, while loops, and output statements only, the technique is limited and cannot be used in general. In particular, it cannot be applied to object-oriented programs.

Laski and Szermer present an algorithm that analyzes corresponding control-flow graphs of the original and modified versions of a program [7]. Their algorithm localizes program changes into clusters, which are single entry, single exit parts of code. Clusters are reduced to single nodes in the two graphs, and then these nodes are recursively expanded and matched. As we discussed in Section 2.4.3, our algorithm is based on this algorithm. However, we make several modifications to the algorithm to improve matching capability (e.g., matching hammocks at different nesting levels, hammock similarity metric, and threshold). In Study 3, we show how our algorithm outperforms, for the case considered, Laski and Szermer's approach in terms of effectiveness in matching two programs.

A recent survey on software merging [8] categorized existing merging techniques. Because differencing approaches are used in the context of software merging, the survey also reported several structure-based differencing approaches and tools used for merging, such as LTDIFF (used in ELAM [5]). These approaches are based on variants of tree-differencing algorithms that operate on programs' parse trees and have some advantages over purely text-based approaches, such as diff. However, these approaches are still limited when used for object-oriented code. For example, these structure-based algorithms work at a finer granularity and can recognize the type of each word in the program text, but they cannot identify the differences in behavior caused by dynamic bindings. According to the categorization pre- 
sented in the survey, JDIFF is a semantic, state-based approach because it captures the changes in program behavior and uses only the information in the original and modified versions of the program.

Although not directly related to this work, Reference [12] presents an interesting study of the type of changes that occur in object-oriented systems during maintenance. Their results confirm that changes in such systems often involve changes in the behavior of object-oriented constructs that must be suitably handled.

\section{Conclusion}

In this paper, we presented an algorithm for comparing two Java programs. The algorithm is based on a methodlevel representation that models the object-oriented features of the language. Given two programs, our algorithm identifies matching classes and methods, builds our representation for each pair of matching methods, and compares the representation for the two methods to identify similarities and differences. The results of our differencing can be used for various development and maintainance tasks, such as impact analysis and regression testing.

We also presented a tool that implements our technique (JDIFF), and a set of studies that show the effectiveness and efficiency of the approach. Study 1 shows how our differencing technique can be successfully applied to coverage estimation. Study 2 illustrates the efficiency of the technique for different execution parameters. Study 3 compares our technique to the most closely related technique and shows that our technique achieves improvements from $17 \%$ to over $65 \%$ in terms of matching unchanged parts of the code.

In future work, we will investigate additional heuristics to further improve the matching results. Our initial experiences in this direction show that there are a number of tradeoffs, in terms of execution cost versus precision, that can be leveraged. We will also study typical changes in evolving systems to assess whether the differencing algorithm could utilize known change patterns. Finally, we will investigate the use of our differencing algorithm to perform test suite augmentation-selecting new test cases for a modified system based on the types of changes performed on the system.

\section{Acknowledgments}

This work was supported in part by National Science Foundation awards CCR-0306372, CCR-0205422, CCR9988294, CCR-0209322, and SBE-0123532 to Georgia Tech, and by the State of Georgia to Georgia Tech under the Yamacraw Mission. Kathy Repine implemented a preliminary version of jdiff. The anonymous reviewers provided useful comments that helped improve the quality of the paper.

\section{References}

[1] S. Elbaum, D. Gable, and G. Rothermel. The impact of software evolution on code coverage information. In Proceedings of the International Conference on Software Maintenance, pages 169-179, Florence, Italy, November 2001.

[2] J. Ferrante, K. J. Ottenstein, and J. D. Warren. The program dependence graph and its use in optimization. ACM Transactions on Programming Languages and Systems, 9(3):319-349, July 1987.

[3] M. J. Harrold, J. A. Jones, T. Li, D. Liang, A. Orso, M. Pennings, S. Sinha, S. A. Spoon, and A. Gujarathi. Regression test selection for java software. In Proceedings of the ACM Conference on Object-Oriented Programming, Systems, Languages, and Applications, pages 312-326, November 2001.

[4] S. Horwitz. Identifying the semantic and textual differences between two versions of a program. In Proceedings of the ACM SIGPLAN'90 Conference on Programming Language Design and Implementation, pages 234-246, White Plains, NY, June 1990.

[5] J. J. Hunt and W. F. Tichy. Extensible language-aware merging. In Proceedings of the International Conference on Software Maintenance, pages 511-520, Montreal, Quebec, Canada, October 2002.

[6] D. Jackson and D. A. Ladd. Semantic diff: A tool for summarizing the effects of modifications. In Proceedings of the Internation Conference on Software Maintenance, pages 243-252, Victoria, B.C., September 1994.

[7] J. Laski and W. Szermer. Identification of program nodifications and its applications in software maintenance. In Proceedings of IEEE Conference on Software Maintenance, pages 282-290, Orlando, FL, November 1992.

[8] T. Mens. A state-of-the-art survey on software merging. IEEE Transasctions on Software Engineering, 28(5):449-462, May 2002.

[9] E. W. Myers. An O(ND) difference algorithm an its variations. Algorithmica, 1(2):251-266, 1986.

[10] A. Orso, T. Apiwattanapong, and M. J. Harrold. Leveraging field data for impact analysis and regression testing. In Proceedings of the European Software Engineering Conference and ACM SIGSOFT Symposium on the Foundations of Software Engineering, Helsinki, Finland, September 2003.

[11] A. Orso, N. Shi, and M. J. Harrold. Scaling regression testing to large software systems. In Proceedings of the ACM SIGSOFT Symposium on the Foundations of Software Engineering, Newport Beach, CA, October 2004 (to appear).

[12] X. Ren, F. Shah, F. Tip, B. G. Ryder, O. Chesley, and J. Dolby. Chianti: A prototype change impact analysis tool for java. Technical Report DCS-TR-533, Department of Computer Science, Rutgers University, September 2003.

[13] G. Rothermel and M. J. Harrold. A safe, efficient regressing test selection technique. ACM Transactions on Software Engineering and Methodology, 6(2):173-210, April 1997.

[14] S. Sinha and M. J. Harrold. Analysis and testing of programs with exception handling constructs. IEEE Transaction on Software Engineering, 26(9):849-871, September 2000.

[15] Z. Wang, K. Pierce, and S. McFarling. BMAT - a binary matching tool for stale profile propagation. The Journal of Instruction-Level Parallelism, 2, May 2000. 